\title{
The Inflativerse - The University of Nottingham's inflatable planetarium
}

\author{
J. R. Ownsworth $\dagger$, B. Haeussler ${ }^{1} \ddagger$, E. Johnston $\uparrow$ and N. Hatch $\|$ \\ University of Nottingham, School of Physics and Astronomy, Nottingham, NG7 2RD, U.K.
}

The Inflativerse is a mobile planetarium run by volunteers lead by the Physics and Astronomy departments at the University of Nottingham. This is a new project set up in 2011 to offer free of charge planetarium shows and activities for schools and public events.

Within this first year of running we have assembled a wide range of Astronomy related talks and activities targeted at an age range of $7-16$. These include talks to younger children about the myths and legends behind the constellations from a variety of different cultures to more advanced talks on why the stars are different colours. It is also possible to talk about subjects outside of astronomy such as ocean currents and plate tectonics. Along side the talks that take place inside the planetarium we also run activities outside of the planetarium. Such activities include planet recognition exercises in the form of a solar system memory game for younger children and using balls and marbles to visualise the scales of sizes and distances in the solar system.

The school visit program is targeted at under performing schools in the local area. These are mostly schools that cannot afford the commercial travelling planetariums and other such education enrichment schemes. This program has been very successful within the first year of operation. We have visited 12 different schools and provided talks and activities to over 1200 children in that time. In this program we offer half and full day visits providing the chance for many children to participate in the activities that we run. We offer this service free of charge to the school.

We also run a public talk program that caters for public events in the local area. This program is for people of all ages to attend. This normally entails a series of talks throughout the event and a team of people outside of the dome to take any questions that the public may want to ask. In our first year we have attending 3 public events including the BBC Stargazing Live. In these events we were able to host over 700 people attending talks within the planetarium.

These programs have been very well received by schools and the public alike and we hope to continue this success into the future. We are now starting to assemble a wide range of activities both in and out of the planetarium and we hope to continually expand this range as time goes on. We are happy to share these activities and receive any ideas for new activities from the wider outreach community.

$\dagger$ E-mail: ppxjo1@nottingham.ac.uk

$\ddagger$ E-mail: boris.haeussler@nottingham.ac.uk

9 E-mail: ppxej@nottingham.ac.uk

|| E-mail: nina.hatch@nottingham.ac.uk 